\title{
Does concurrent adenoidectomy or tonsillectomy affect the graft success rate of cartilage myringoplasty in adults?
}

Zhengcai Lou* (1)

\begin{abstract}
Background: The objective of this study was to evaluate the graft success and hearing outcomes of concurrent adenoidectomy or tonsillectomy and myringoplasty.

Methods: Medical case notes were reviewed for all adult patients with dry perforations who had undergone myringoplasty, with or without concurrent throat surgery, from December 2015 to February 2018. The study population was divided into concurrent myringoplasty and throat surgery (Group A) and single myringoplasty (Group B) groups. The air-bone gap (ABG) and graft success rate were evaluated in both groups.

Results: A total of 131 ears of 131 patients were included in this study. In total, 33 ears of 33 patients were assigned to Group A and 98 to Group B. Of the 33 patients in Group A, adenoid residue was detected in 3, chronic tonsillitis in 21 , and tonsil hypertrophy in 9. The graft success rate was $96.9 \%$ in Group A and $96.9 \%$ in Group B at 6 months postoperatively ( $p=0.993)$. In addition, the graft success rate was $87.9 \%$ in Group A and $92.8 \%$ in Group B at 24 months postoperatively ( $p=0.372$ ). Reperforation occurred in three patients in Group A and four in Group B; the difference was not significant. No significant group differences were observed in preoperative $(p=0.654)$ or postoperative $(p=0.791) A B G$ values or mean ABG gain $(p=0.439)$. No patient in either group developed cholesteatoma of the middle ear.
\end{abstract}

Conclusions: Simultaneous adenoidectomy or tonsillectomy and myringoplasty is feasible but does not improve the graft success rate or hearing outcome.

Keywords: Endoscope, Myringoplasty, Adenoidectomy, Tonsillectomy, Graft success

\section{Background}

Chronic tympanic membrane (TM) perforation with chronic otitis media $(\mathrm{COM})$ are related to inadequate ventilation through the eustachian tube (ET) [1,2]. Adult patients with chronic otologic disease frequently have coexisting nasal and throat pathology, which can cause or worsen middle ear problems secondary to eustachian tube dysfunction (ETD) [1]. The causes of ETD include

*Correspondence: louzhengcai@163.com

Department of Otorhinolaryngology, Yiwu Central Hospital, 699

Jiangdong Road, Yiwu 322000, Zhejiang, China upper respiratory tract infection, sinusitis, allergic rhinitis, adenoid, tonsil hypertrophy, nasopharyngeal mass, cleft palate, and nasal septal deviation [1,3-6]. ETD can result in chronic negative middle ear pressure, which can cause TM retraction, COM with effusion, and middle ear infection [2]. Thus, some studies suggested that nasal abnormalities and pathology should be identified and corrected before myringoplasty is performed [7, 8]. Sinonasal and nasopharyngeal procedures may improve ET function and, by extension, otologic outcomes [1].

Whether simultaneous ear and nasal/sinus procedures should be performed is debated. Two studies suggested 
that simultaneous myringoplasty and septoplasty is feasible in adults with both middle ear and sinonasal pathology, and is attractive in terms of operative and anesthetic morbidity, time, and the lower cost of surgically repairing nasal and ear problems simultaneously $[1,9]$. However, others disapproved of simultaneous nasal surgery and myringoplasty because it increases the risk of graft reperforation [7, 10, 11]. Salvinelli et al. [12] recommended that tympanoplasty and nasal surgery not be performed at the same time, and that middle ear surgery should be carried out when the anatomy and physiology of the nasal, pharyngeal, and tubal mucosae have returned to normal. In children, Becker and Opitz [13] also concluded that adenoidectomy should not be performed concurrently with tympanoplasty because of frequent postoperative negative middle ear pressure. Charlett et al. [14] suggested that adenoidectomy before pediatric myringoplasty does not increase the likelihood of a successful outcome. However, few studies have evaluated the effect of throat disorders on the success of myringoplasty in adults. We evaluated the graft success rate and hearing outcome of concurrent adenoidectomy or tonsillectomy and myringoplasty.

\section{Methods}

\section{Patients and methods}

Medical case notes were reviewed for all adult patients with dry perforations who had undergone myringoplasty, with or without concurrent throat surgery, from December 2015 to February 2018 in a single teaching hospital. Exclusion criteria were the presence of cholesteatoma, malignant laryngeal tumors, revision cases, procedures involving ossicular reconstruction or mastoid surgery, history of previous adenoidectomy or tonsillectomy, and failure to attend postoperative follow-up. All operations were performed by the same surgeon.The perforation was classified according to size, as large ( $>50 \%$ of the eardrum), medium $(25-50 \%)$, or small $(<25 \%)$.

The study population was divided into concurrent myringoplasty and throat surgery (Group A) and single myringoplasty (Group B) groups. The throat surgery group comprised patients who had a concurrent adenoidectomy or tonsillectomy. Data on age, sex, side, size of perforation, myringosclerosis, smoking status, followup duration, audiologic test results, TM graft status at the most recent follow-up, and surgical outcomes were obtained from the patient's medical charts. Pure-tone audiometry (PTA) was performed preoperatively and at 12 months after surgery. Standard PTA was performed at the frequencies of $0.5,1,2$, and $3 \mathrm{kHz}$. The air-bone gap (ABG) was calculated as the mean difference between air conduction and bone conduction at each frequency.

\section{Surgical techniques}

\section{Concurrent myringoplasty and throat surgery (Group A)}

All patients were scheduled for simultaneous myringoplasty and tonsillectomy or adenoidectomy under general anesthesia. Same-day myringoplasty was performed in patients with bilateral TM perforations. The pharyngeal surgery was performed before myringoplasty. Tonsillectomies were performed using monopolar electrosurgery in patients with tonsil hypertrophy (Fig. 1), and plasma radiofrequency ablation was used for adenoidectomy. All tissue samples were sent for histological examination.

Following the tonsillectomy or laryngeal surgery, myringoplasty using a full-thickness cartilage graft was performed under a $0^{\circ}, 4 \mathrm{~mm} \cdot 18 \mathrm{~cm}$ rigid endoscope using the "push-through" technique. Cartilage from a single-layer perichondrium graft was harvested through a 1-cm incision medial to the ipsilateral tragus. The graft was 1-2 mm wider than the diameter of the perforation and was not thinned. If the malleus was exposed, a notch was created in the cartilage (but not the perichondrium) to accommodate the handle. The perichondrium lateral to the notch was peeled and elevated, thus becoming a patch of free perichondrium $2 \mathrm{~mm}$ wider than the notch. The composite graft was pushed through the perforation and placed medial to the remnant TM and the annulus in an underlay manner. Then, the cartilage notch was clipped to the malleus, and the patch of free perichondrium above the notch was placed lateral to the handle of the malleus. The tympanomeatal flap was not elevated in

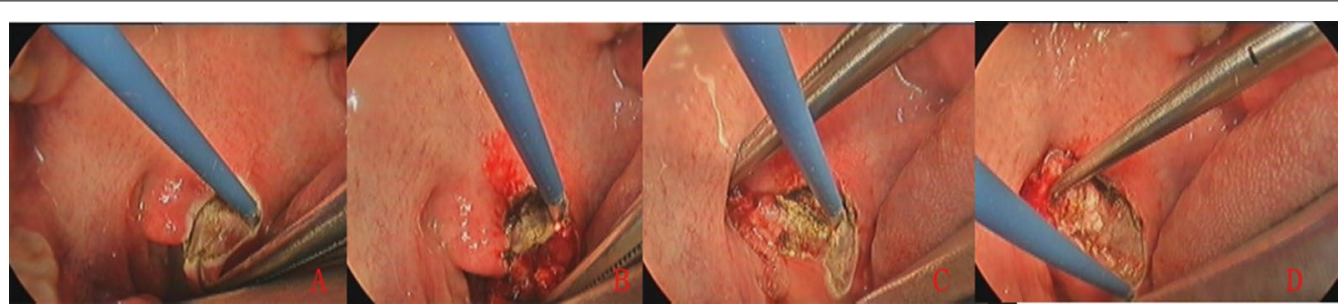

Fig. 1 Photographs showing the upper pole of the tonsil (A and $\mathbf{B})$, tonsils separated in peritonsillar space (C), and dissection of the inferior pole of the tonsil (D) 
any patient. Biodegradable Nasopore soaked in antibiotic ointment was used to support the graft medially and laterally. The external auditory canal was packed with gauze soaked in antibiotic ointment up to the tragus incision, which was not sutured.

\section{Single myringoplasty group (Group B)}

Single myringoplasty without tonsillectomy or adenoidectomy was performed. The surgical procedure for myringoplasty was similar to that for Group A.

\section{Postoperative follow-up}

The patients were discharged after 2 days. The packing gauze soaked in antibiotic ointment was removed from the EAC at 2 weeks postoperatively, and the biodegradable Nasopore fragments were aspirated from the EAC at 3 weeks after surgery to allow visualization of the graft. All patients were scheduled for regular follow-up visits at 2 and 3 weeks and $1,3,6,12$, and 24 months after surgery in the otolaryngology outpatient clinic. Endoscopy was performed at all postoperative visits, and PTA was performed at the 12-month follow-up visit. Graft success was defined as the presence of an intact graft, as evaluated using a $0^{\circ}$ endoscope. Graft failure was defined as residual perforation, recurrent perforation, graft lateralization, significant blunting, and medialization at 6 and 24 months postoperatively.

\section{Statistical analysis}

Statistical analyses were performed using statistic package for social science (SPSS) software ver. 21.0; (SPSS Inc.,, Chicago, IL, USA). Data are expressed as means with standard deviations, or as percentages (\%). The chisquared test was used to compare categorical data. The Wilcoxon and Mann-Whitney $U$ tests were employed to compare non-parametric variables, and the independent and paired samples $t$-tests were used to compare parametric variables. A p-value $<0.05$ was considered to indicate statistical significance.

\section{Results}

\section{Demographic data}

In total, 131 ears of 131 patients met the inclusion criteria. Of them, 33 ears of 33 patients were assigned to the concurrent myringoplasty and throat surgery group (Group A), and 98 ears to the single myringoplasty group (Group B). Of the 33 patients in Group A, adenoid residue was detected in 3 patients (Fig. 2), chronic tonsillitis in 21, and tonsil hypertrophy in 9 (Fig. 1). The age, sex, side, type, size of perforation, myringosclerosis, and smoking status were matched between the two groups (Table 1). Postoperative pathology tests confirmed tonsil hypertrophy or adenoid in all cases in Group A. No evidence of postoperative bleeding was found.

\section{Graft uptake rate and complications}

All patients were followed up for 24 months. The graft success rate was $96.9 \%(32 / 33)$ in Group A and $96.9 \%(95 / 98)$ in Group B at 6 months postoperatively $(\mathrm{p}=0.993)$ (Fig. 3). In Group A, one ear had postoperative

Table.1 Demographic characteristic of patients between the groups

\begin{tabular}{llll}
\hline & A group & B group & P value \\
\hline No & 33 & 98 & \\
Sex (F:M) & $11: 22$ & $41: 57$ & $0.388^{\text {a }}$ \\
Age (years) & $47.6 \pm 3.76$ & $49.3 \pm 4.17$ & $0.573^{\mathrm{b}}$ \\
Side of ear (L:R) & $20: 13$ & $69: 29$ & $0.297^{\mathrm{a}}$ \\
Size of perforation (Large: & $9: 22: 2$ & $28: 59: 11$ & $0.391^{\mathrm{a}}$ \\
$\quad$ Medium:Small) & & & \\
Type of perforation (marginal:central) & $14: 19$ & $41: 57$ & $0.953^{\mathrm{a}}$ \\
Myringosclerosis (Y:N) & $10: 23$ & $27: 71$ & $0.761^{\text {a }}$ \\
Smoking status (Y:N) & $7: 26$ & $16: 82$ & $0.523^{\mathrm{a}}$ \\
Graft success rate & & & \\
$\quad$ At postoperative 6 ${ }^{\text {th }}$ months (N,\%) & $32(96.9 \%)$ & $95(96.9 \%)$ & $0.993^{\mathrm{a}}$ \\
$\quad$ At postoperative 24th months (N,\%) & $29(87.9 \%)$ & $91(92.8 \%)$ & $0.372^{\mathrm{a}}$ \\
$\quad$ Re-perforation (N, \%) & $3(9.4 \%)$ & $4(4.21 \%)$ & $0.268^{\mathrm{a}}$ \\
\hline a Chi-square test & & & \\
${ }^{\mathrm{b}}$ Independent Samples Test & & &
\end{tabular}

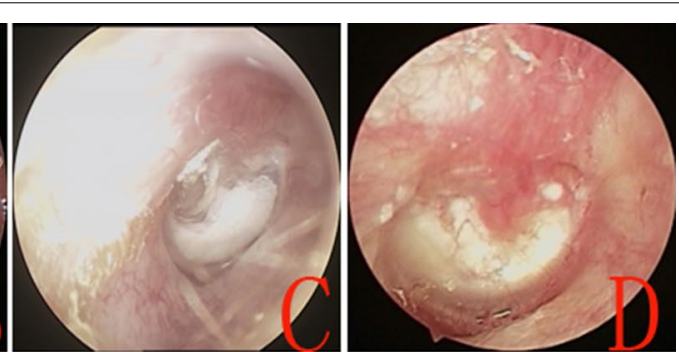

Fig. 2 A-29-year-old male with right TM perforation and coexistent adenoid residue. The adenoid residue preoperatively (A), the nasopharynx at 2 weeks postoperatively (B), te preoperative perforation (C), and perforation closure at 3 months postoperatively (D) 


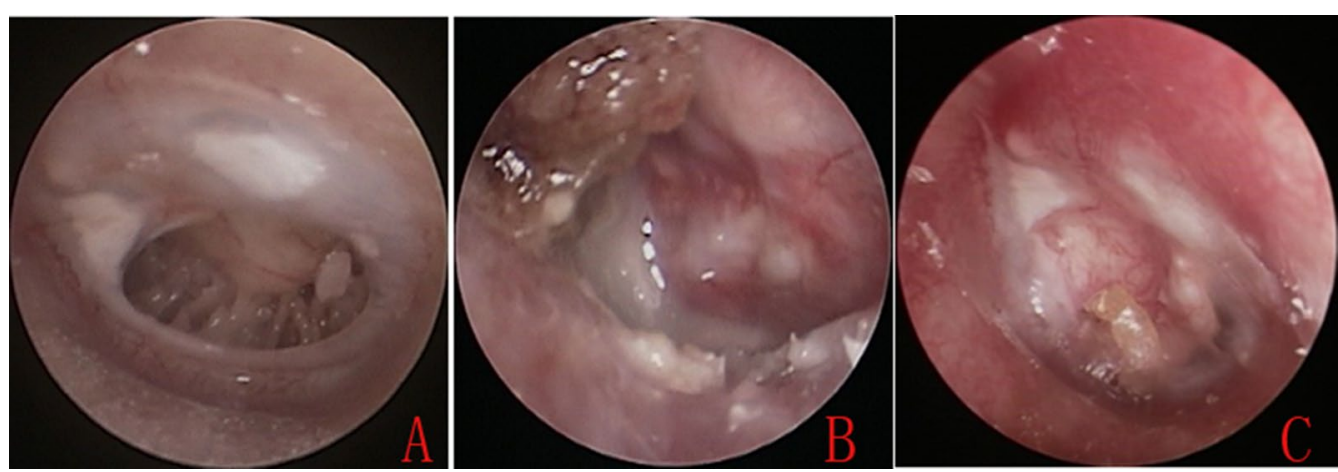

Fig. 3 Photographs showing the perforation before surgery (A), and at 2 weeks (B) and 5 weeks (C) post-surgery (same patient as in Fig. 1)

purulent otorrhea and secondary middle ear infection, resulting in residual perforation. In Group B, residual perforation was seen in three patients. The graft success rate was $87.9 \%(29 / 33)$ in Group A and $92.8 \%(91 / 98)$ in Group B at 24 months postoperatively $(\mathrm{p}=0.372)$. Reperforation occurred in three patients in Group A and four patients in Group B $(\mathrm{p}=0.268)$ (Table 1). During followup, no adenoidectomy- or tonsillectomy-related complications were observed. No complications (iatrogenic sensorineural hearing loss, facial nerve palsy, vertigo, or tinnitus) were observed, and no graft lateralization or medialization, or significant blunting was noted. No patients in either group developed cholesteatoma of the middle ear.

\section{Hearing gain}

In Group A, the mean pre- and postoperative ABGs were $26.83 \pm 4.15$ and $9.61 \pm 2.54 \mathrm{~dB}$ at 12 months postoperatively $(p=0.001)$. In Group $B$, the values were $27.31 \pm 6.91 \mathrm{~dB}$ and $8.74 \pm 3.61 \mathrm{~dB}(\mathrm{p}=0.001)$. No significant group differences were observed in the preoperative $(p=0.654)$ or postoperative $(p=0.791)$ ABG values or mean ABG gain $(\mathrm{p}=0.439)$ (Table 2$)$. The functional success rate (postoperative $A B G \leq 20 \mathrm{~dB})$ was $84.8 \%(n=28)$ in Group A and $87.8 \%(n=86)$ in Group B $(\mathrm{p}=0.769)$.

\section{Discussion}

ETD and the consequent hypoventilation of the middle ear are among the most frequent causes of failure of middle ear surgery. However, nasal or pharyngeal pathology is often thought to be responsible for inadequate tubal function. Therefore, potential interactions among the middle ear mucosa, ET function, and nasal or pharyngeal pathology are considered when planning myringoplasty $[7,8]$. Several sinonasal and nasopharyngeal procedures can enhance tubal function and thereby improve otologic outcomes $[8,15,16]$. In addition, performing an adenoidectomy before TM reconstruction would improve graft survival rates for patients with adenoidal or tonsil hypertrophy [14]. However, the timing of sinonasal or nasopharyngeal procedures and myringoplasty are controversial. In most studies, myringoplasty was performed prior to a sinonasal or nasopharyngeal procedure, because transient tubal dysfunction and negative middle ear pressure can result in graft failure $[7,10]$. However, in two studies simultaneous nasal surgery and myringoplasty did not affect the graft success rate $[1,9$. Similarly, some studies reported frequent negative middle ear pressure in children following adenoidectomy, and this procedure should not be performed concurrently with tympanoplasty $[13,14]$.

Table 2 Comparison of hearing gains and the air-bone gap ((dB) mean $\pm S D$ )

\begin{tabular}{|c|c|c|c|c|c|}
\hline & Pre-ABG & Post- ABG & $P^{1}$ & Gain (mean) & $\begin{array}{l}\text { Hearing } \\
\text { success } \\
(A B G \leq 20 \mathrm{~dB})\end{array}$ \\
\hline Group A $(n=33)$ & $26.83 \pm 4.15$ & $9.17 \pm 2.26$ & $0.001 *$ & $17.41 \pm 5.64$ & $28(84.8 \%)$ \\
\hline Group B $(n=98)$ & $27.31 \pm 6.91$ & $8.74 \pm 3.61$ & $0.001^{*}$ & $19.31 \pm 2.31$ & $86(87.8 \%)$ \\
\hline$p^{2}$ & 0.654 & 0.791 & & 0.439 & $0.769^{a}$ \\
\hline
\end{tabular}

\footnotetext{
${ }^{1}$ Paired Samples test, ${ }^{2}$ Mann Whitney $\mathrm{U}$ test, ${ }^{\mathrm{a}} \mathrm{Chi}$-square test

*p $<0.01$

${ }^{1}$ Comparison ABG between the same groups pre- and postoperatively

${ }^{2}$ Comparison between two groups in terms of gain, pre- or postoperatively
} 
in this study, the graft success rate was $96.9 \%(32 / 33)$ in Group A and 96.9\% (95/98) in Group B at 6 months postoperatively $(\mathrm{p}=0.993)$ (Fig. 3). In Group A, one ear had postoperative purulent otorrhea and secondary middle ear infection, resulting in residual perforation. In Group B, residual perforation was seen in three patients. The graft success rate was $87.9 \%(29 / 33)$ in Group A and $92.8 \%$ (91/98) in Group B at 24 months postoperatively $(\mathrm{p}=0.372)$. No significant group differences were observed in the preoperative $(\mathrm{p}=0.654)$ or postoperative $(\mathrm{p}=0.791)$ ABG values or mean ABG gain $(\mathrm{p}=0.439)$.

Our data indicate that simultaneous myringoplasty and throat surgery is efficacious in terms of TM graft survival and overall surgical success. These results are in agreement with Schuman, who concluded that simultaneous tympanoplasty and nasal surgery is feasible in adults [1]. Also, simultaneous surgery is attractive in terms of operative and anesthetic morbidity, time, and cost.

Adenoidectomy and tonsillectomy did not improve the graft success rate in patients with adenoid residue and tonsil hypertrophy, respectively, as reported previously in children [13, 14]. Charlett et al. [14] found that adenoidectomy before pediatric myringoplasty may not improve the likelihood of a successful outcome. Interestingly, Vartiainen et al. [17] performed a retrospective study of 60 pediatric patients with dry TM perforation undergoing type I tympanoplasty and found that all failures occurred in patients who had previously undergone adenoidectomy or adenotonsillectomy [17]. One possible explanation for this is that long-term adenoid or tonsillar hypertrophy resulted in morphological changes and irreversible stenosis of the cartilaginous part of the ET, but not of the edema of the mucous membrane at the tubal orifice. This precludes normalization of ET morphology even if the adenoid or tonsil is removed. Becker et al. [13] reported that most ET functions had not returned to normal following adento-tonsillectomy in children. In addition, rhinoplasty did not improve the function of the ET or the outcome of myringoplasty $[7,18,19]$.

Although passive tubal parameters showed considerable improvement in many patients, there was no clear improvement of active tubal parameters following nasal surgery in most patients [7]. In contrast, coexisting chronic sinusitis is the factor most significantly associated with graft failure and reperforation [20]. Therefore, it has been suggested that sinonasal and nasopharyngeal procedures are useful for improving ET function in cases with chronic nasal or nasopharyngeal infection, if accompanied by poor tubal function [7]. A possible mechanism is direct inflammation arising from chronic infection at the tubal orifice on the mucous membrane. Sinusitis, and its irritant effect on the tubal mucous membrane, may resolve following surgery [8]. Other studies reported that negative middle ear pressure is related to graft retraction rather than failure [8]. In addition, cartilage grafts are stiff and can easily withstand negative middle ear pressure, which may have contribute to the development of otitis media and significantly affect postoperative healing outcomes [21, 22]. Therefore, cartilage grafts may prevent changes in ET function after myringoplasty.

This study was limited by the lack of assessment of preoperative and postoperative ET function. Also, this was not a randomized controlled trial, and multivariate analysis was not performed to identify risk factors for graft failure.

\section{Conclusions}

Concurrent adenoidectomy or tonsillectomy and myringoplasty is feasible but does not improve the graft success rate or hearing outcome.

\section{Abbreviations \\ TM: Tympanic membrane; COM: Chronic otitis media; ET: Eustachian tube; ETD: Eustachian tube dysfunction; ABG: Air-bone gap; SPSS: Statistic package for social science.}

\section{Acknowledgements \\ Not applicable.}

\section{Authors' contributions}

LZC conceived and designed the study, acquired and interpreted the data, and drafted. The author read and approved the final manuscript.

\section{Funding}

This study was supported by the Health Commission of Zhejiang Province, China (Grant \#2021KY1186) and the Science and Technology Agency of Yiwu City, China (Grant \#2018-3-76).The funding supported the design of the study, the collection, analysis, and interpretation of the data, and the writing of the manuscript.

\section{Availability of data and materials}

All data generated or analyzed during this study are included in the published article.

\section{Declarations}

\section{Ethics approval and consent to participate}

The study protocol was approved by the Human Research Ethics Committee of Yiwu Central Hospital, which adheres to local policy, national laws, and the World Medical Association Declaration of Helsinki. Written informed consent was obtained from all patients.

\section{Consent for publication}

Written informed consent was obtained from all patients for publication of this study and any accompanying images. A copy of the written consent form is available for review by the Editor-in-Chief of this journal.

\section{Competing interests}

The authors declare that they have no competing interests.

Received: 16 November 2020 Accepted: 1 June 2021

Published online: 08 June 2021 


\section{References}

1. Schuman TA, Labadie RF. Concurrent nasal surgery and tympanoplasty in adults. Ear Nose Throat J. 2010;89:E28-32.

2. Seibert JW, Danner CJ. Eustachian tube function and the middle ear. Otolaryngol Clin North Am. 2006;39(6):1221-35.

3. Higgins TS, Cappello ZJ, Wu AW, Ting JY, Sindwani R. Predictors of eustachian tube dysfunction improvement and normalization after endoscopic sinus surgery. Laryngoscope. 2019. https://doi.org/10.1002/lary. 28416.

4. Bowles PFD, Agrawal S, Salam MA. Eustachian tube dysfunction in chronic rhinosinusitis: pre and post-operative results following endoscopic sinus surgery, a prospective study. Rhinology. 2019;57:73-7.

5. Juszczak H, Aubin-Pouliot A, Sharon JD, Loftus PA. Sinonasal risk factors for eustachian tube dysfunction: Cross-sectional findings from NHANES 2011-2012. Int Forum Allergy Rhinol. 2019;9:466-72.

6. tilla MH, Kaytez SK, Kesici GG, Baştimur S, Tuncer S. Comparison between curettage adenoidectomy and endoscopic-assisted microdebrider adenoidectomy in terms of Eustachian tube dysfunction. Braz J Otorhinolaryngol. 2020;86(1):38-43.

7. Maier W, Krebs A. Is surgery of the inner nose indicated before tympanoplasty? Effects of nasal obstruction and reconstruction on the Eustachian tube. Laryngorhinootologie. 1998;77:682-8.

8. Grady D, Mathias P, Anderson R, Snider G, Sprinkle PM. Improvement of middle ear disease following septoplasty. Am J Otol. 1983:4(4):327-31.

9. Uçar C, Kazkayasi M. Simultaneous myringoplasty and septoplasty, and the use of nasal septal perichondrium. Eur Arch Otorhinolaryngol. 2009;266(8):1213-7.

10. Laszig R. Development of pressure in the middle ear after nasal operations. HNO. 1985;33(4):187-9 German.

11. Koch U, Herberhold C, Opitz HJ. [Middle ear pressure after rhinoplasty surgery (author's transl)]. Laryngol Rhinol Otol (Stuttg). 1977;56(8):657-61. German.

12. Salvinelli F, Casale M, Greco F, D'Ascanio L, Petitti T, Di Peco V. Nasal surgery and eustachian tube function: effects on middle ear ventilation. Clin Otolaryngol. 2005;30(5):409-13.
13. Becker W, Opitz HJ. The problems of simultaneous tympanoplasty and adeno-tonsillectomy [author's transl; in German]. Laryngol Rhinol Otol (Stuttg). 1978;57(3):203-9.

14. Charlett SD, Knight LC. Pediatric myringoplasty: does previous adenoid ectomy improve the likelihood of perforation closure? Otol Neurotol. 2009;30:939-42.

15. Metson R, Pletcher SD, Poe DS. Microdebrider eustachian tuboplasty: A preliminary report. Otolaryngol Head Neck Surg. 2007;136(3):422-7.

16. Poe DS, Grimmer JF, Metson R. Laser eustachian tuboplasty: Two-year results. Laryngoscope. 2007;117(2):231-7.

17. Vartiainen E, Vartiainen J. Tympanoplasty in young patients: the role of adenoidectomy. Otolaryngol Head Neck Surg. 1997;117:583-5.

18. Akyıldız MY, Özmen ÖA, Demir UL, Kasapoğlu F, Coşkun HH, Basut O, et al. Should nasal function be considered prior to tympanoplasty? J Int Adv Otol. 2018;14(1):53-7.

19. Eser B, Yılmaz A, Önder S, Toros SZ, Oysu Ç. The effect of nasal functions on the integrity of grafts after myringoplasty. Turk Arch Otorhinolaryngol. 2017;55:153-7.

20. Jesic SD, Dimitrijevic MV, Nesic VS, Jotic AD, Slijepcevic NA. Temporalis fascia graft perforation and retraction after tympanoplasty for chronic tubotympanic otitis and attic retraction pockets: factors associated with recurrence. Arch Otolaryngol Head Neck Surg. 2011;137:139-43.

21. Chhapola S, Matta I. Cartilage-perichondrium: an ideal graft material? Indian J Otolaryngol Head Neck Surg. 2012;64:208-13.

22. Huang YB, Hu LL, Ren DD, Han Z. Myringoplasty with an ultrathin cartilage-perichondrium complex graft versus temporalis fascia graft: a propensity score-matched analysis. Otolaryngol Head Neck Surg. 2020;164(6):1287-93.

\section{Publisher's Note}

Springer Nature remains neutral with regard to jurisdictional claims in published maps and institutional affiliations.
Ready to submit your research? Choose BMC and benefit from:

- fast, convenient online submission

- thorough peer review by experienced researchers in your field

- rapid publication on acceptance

- support for research data, including large and complex data types

- gold Open Access which fosters wider collaboration and increased citations

- maximum visibility for your research: over $100 \mathrm{M}$ website views per year

At $\mathrm{BMC}$, research is always in progress.

Learn more biomedcentral.com/submissions 